\title{
Del problema de la muerte al misterio de la muerte en Gabriel Marcel
}

\section{From Problem of Death to Mystery of Death in Gabriel Marcel}

\author{
YEFREY A. RAMÍREZ*
}

\begin{abstract}
Resumen: Ante las numerosas amenazas a las que está sujeto el hombre, la proximidad de la muerte puede hacer declinar frente a la vida provocando un estado de desesperación por la posibilidad de destrucción de todo lo que se ha construido día tras día. Frente a ello, Gabriel Marcel, filósofo francés, encuentra en las relaciones humanas una nueva forma de comprensión de la muerte y, por ende, de la existencia. A través de la relación con el otro, especialmente con el amado, tomó conciencia del ser que nos subyace. El reconocimiento del ser como exigencia ontológica y de la libertad como acto que permite ser lo que soy en base a la intersubjetividad, libra al hombre de la desesperación. Para comprender ello, hay que pensar la muerte no desde la reflexión primera como problema, sino desde la reflexión segunda como misterio.

Palabras claves: Muerte, ser, intersubjetividad, libertad, exigencia ontológica.
\end{abstract}

\begin{abstract}
Death's closeness, by the numerous threats to which man is subject, could lead us to lose the will to live as it causes a state of despair when we see all the things that we have built every day sentenced to destruction. In light of this, French philosopher Gabriel Marcel finds a new way to understand the existence and death inhuman relationships. Through one's relationship with the other, especially with the loved one, one becomes aware of the being that lies underneath us. The recognition of the being like demand of being and freedom as an act that allow us, based on intersubjectivity, be who we are, free to men of the despair. To understand that, it's necessary to think about death not from first reflection as a problem, but rather from second reflection as a mystery.
\end{abstract}

Key's words: Death, being, intersubjectivity, freedom, demand of being.

\section{Introducción ${ }^{1}$}

La muerte ha sido un tema recurrente en pensadores de corte existencial. Gabriel Marcel, filósofo parisino del siglo pasado, mostró también un interés particular por este tópico desde sus primeras obras. No obstante, pocos trabajos se han dedicado a resaltar esta predilección ${ }^{2}$,

\section{Recibido: 08/02/2017. Aceptado: 06/10/2018.}

* Doctor en Filosofía por la Universidad de Salamanca con la tesis titulada "La muerte en Gabriel Marcel". Autor del artículo "El sufrimiento en Gabriel Marcel”, en Estudios Filosóficos, Vol. LXVI, n 193, pp. 525-242. Correo electrónico: yefrey12@gmail.com, Universidad de Salamanca.

1 Una primera versión de este artículo apareció en la revista Nova et Vetera con el título: "La muerte en Gabriel Marcel: metafísica y gnoseología”, No 83, año XLI, enero-junio 2017, pp. 93-109.

2 De entre ellos se puede citar ANDERSON (2006), GARCÍA (1974), HANLEY (1976), LONERGAN (1975), RIGOL (2014), RÍOS (1999), RUIZ (2016), URABAYEN (2001). Sobre la muerte en la obra dramática de Marcel: BELAY (1980), MAYO (1978). 
si bien ésta ha sido esencial para el desarrollo del pensamiento marceliano. De hecho, el suceso de la muerte le despertó el atractivo por la filosofía cuando siendo apenas un niño preguntó a su tía por los muertos y, sin haber obtenido una respuesta satisfactoria, pensó que se ocuparía de ello más adelante ${ }^{3}$.

La muerte es el origen de su pensamiento por la razón biográfica nombrada y porque es un referente primordial para el planteamiento de temas claves como el ser, la intersubjetividad, la esperanza y la fidelidad. Del ser porque es el contrapeso a la nada y al absurdo que parece abrirse con la muerte ${ }^{4}$. Y tanto de la intersubjetividad como de la esperanza y la fidelidad porque la muerte es la prueba de las relaciones humanas más profundas ${ }^{5}$. A ellas Marcel fue especialmente sensible desde su niñez, comparándolas con la consonancia y armonía que surgen en la música ${ }^{6}$.

Lo anterior deja saber que solamente desde un enfoque existencial se puede entender la muerte como un referente, por eso Marcel tiene al inicio de su reflexión la existencia humana ${ }^{7}$. Bajo esta óptica, la experiencia es la que brinda los datos de análisis; pero no sólo las experiencias sensoriales, sino las que pueden llamarse interiores por ser las más intensamente vividas ${ }^{8}$. Por eso la necesidad de la distinción entre una reflexión que se dedique al análisis y la descomposición de lo verificable empíricamente, cuyo escrutinio le corresponde a la experiencia sensorial, y otra que tome lo que en ésta hay de vacío o de sospechoso y desarrolle una nueva reflexión recuperadora del hombre concreto, a través de la cual se puedan pensar las demás experiencias.

Desde el método gnoseológico que Marcel especificó, basado en la diferencia entre reflexión primera, que se ocupa de los problemas, y reflexión segunda, que trata sobre los misterios $^{9}$, la cuestión de la muerte adquiere matices que diferencia el tratamiento que se le da a la cuestión sobre "mi muerte" y a la "muerte del ser amado". En un principio Marcel ve serias dificultades al pensar mi muerte desde los presupuestos de la reflexión primera. No obstante, en sus obras de madurez, lleva a sus últimas consecuencias la reflexión segunda sobre "la muerte del ser amado", convirtiéndola en un espejo iluminador sobre la relación que une al hombre concreto con su muerte. Todo ello será lo que se tratará de desglosar en la siguiente exposición.

\section{La muerte y el ser}

El hombre al pensar su existencia, que no es un demostrandum ${ }^{10}$ sino un indubitable anterior al juicio mismo ${ }^{11}$, se encuentra con la cuestión de la no existencia, por cuenta del hecho de la muerte. Este asunto rondó el pensamiento de Marcel a causa de la muerte de su madre

3 Cf. TM, 7; DH, 43; FTC, 42-43. La lista de abreviaturas se encuentra al final del documento.

4 Cf. MEII, 57-60. 147.

5 Cf. EAI, 105; RI, 100-101; HV, 38; RI, 198-199.

6 Cf. ECD, 21.

7 Cf. RI, 26.

8 Cf. RI, 89.

9 Cf. EAI, 144-150.

10 Cf. JM, 32.

11 Marcel considera que no se puede demostrar la existencia en oposición a Descartes, quien utiliza la necesidad de la demostración de la existencia para encontrar un indubitable en la filosofía. Estas ideas las desarrolla en su 
cuando tenía apenas cuatro años y de sus experiencias durante la I Guerra Mundial ${ }^{12}$. Dichas situaciones le hacen preocuparse por la profundidad de las relaciones humanas, conato de lo que sería posteriormente definido como intersubjetividad ${ }^{13}$ ¿ ¿Las relaciones humanas se destruyen absolutamente con la muerte? ¿La muerte tiene la última palabra frente a la existencia? Éstos son asuntos sobre los cuales versan los principales temas de este autor francés: intersubjetividad, fidelidad, esperanza y el misterio ontológico.

Precisamente, Marcel empezó a hablar de ser después de abandonar el idealismo porque le parecía que el hombre del que habla la filosofía post-hegeliana poco podía decir ante lo trágico de la vida, pues ésta versa sobre un ser inmanente e impersonal. Juzgaba, entonces, que desde esas proposiciones y enunciados no se podía hacer una explicación de los problemas existenciales, especialmente cuando la muerte se vuelve una amenaza constante. Esta fue la razón por la que Marcel adoptó una filosofía de corte individual y trascendente ${ }^{14}$, desde la cual, la cuestión de la muerte se descubre como la destrucción, la aniquilación, en última instancia, el no-ser ${ }^{15}$.

Marcel inicia su reflexión sobre el ser, entonces, basado en la experiencia concreta, tema que le causaba repugnancia, sin podérsela justificar ${ }^{16}$. Para él, esa incapacidad para eludir el ser depende, directamente, de la afirmación del principio de identidad a través del cual funciona el pensamiento ${ }^{17}$. A partir de allí, entendió la conciencia como conciencia de algo, ya que el acto de afirmar y enunciar de la conciencia se hace sobre otro o algo distinto de síl ${ }^{18}$. El pensamiento se dirige a otro. Si se niega esto, la conciencia, según Marcel, quedaría volcada en un solipsismo ${ }^{19}$.

Por lo anterior, el pensamiento está dirigido, al modo fenomenológico de Husserl, a las esencias. No obstante, Marcel distingue, a diferencia de este filósofo alemán, dos clases de pensamiento: el pensar y el pensar en: el primero es el que reconoce estructuras en el mundo, mientras que el segundo está volcado hacia el $\operatorname{ser}^{20}$. Por el primero funciona la reflexión primera y lo problemático, mientras que por el segundo lo hace la reflexión segunda y el

conocido ensayo Existencia y objetividad que apareció en la parte final del Journal Métaphysique (JM), 309329.

12 Sobre la muerte de su madre escribió: "No sorprenderé a nadie al decir que es la situación familiar la que debe ser tenida en cuenta, pero desde su origen esta situación debía verse afectada, en cuanto a mí, por un indicio que debo denominar metafísico. El acontecimiento decisivo fue en realidad la muerte de mi madre, que me fue arrebatada en cuarenta y ocho horas, cuando yo iba a cumplir cuatro años". EVJ, 14. Sobre su experiencia en la guerra relató: "Lo que impedía que fuera así -ver la guerra abstractamente- eran las visitas que recibía varias veces al día y que, casi siempre, me conmovían porque me ponían en fde un sufrimiento y una angustia concreta. Y lo que estaba a mi mano era por lo menos acoger a estas gentes que venían a mí de manera lo bastante humana y personal como para que no tuvieran la impresión de dirigirse a una oficina o ventanilla. Creo que esto fue muy importante, porque fue en el fondo como un primer aprendizaje de la intersubjetividad tal como había de definirla más tarde". ECD, 71.

13 Cf. ECD, 74.

14 Cf. HV, 181-182.

15 Cf. FTC, 166; PI, 105.

16 Cf. EAI, 34.

17 Cf. EAI, 32-33.42-45.

18 Cf. MEI, 61.

19 Cf. MEII, 11-12.19-20.

20 Cf. EAI, 36-37. 
misterio. Por esta razón el ser no se puede problematizar, sino que hay que hablar de "misterio del ser".

Dentro de las razones que llevan a Marcel a hacer esta distinción se encuentran las objeciones que Kant hizo sobre el ser. La principal de ellas, que el ser no se puede convertir en un predicado ${ }^{21}$. Para Marcel, esto se explica porque el ser escapa a toda descripción objetiva, pues pertenece al orden del misterio. Ahora bien, que sea un misterio, no quiere decir que sea incognoscible. Sobre esto, este filósofo parisino explica:

Por el contrario, el reconocimiento del misterio es un acto esencialmente positivo del espíritu, el acto positivo por excelencia y en función del cual quizás se defina rigurosamente toda positividad. Todo parece suceder aquí como si yo disfrutara de una intuición que poseo sin saber inmediatamente que la poseo, una intuición que no podría ser propiamente hablando, para sí, pero que no se aprehende a sí misma a través de los modos de experiencia sobre los cuales se refleja y que ella misma ilumina mediante dicha reflexión. La labor metafísica esencial consistirá, entonces, en una reflexión sobre esta reflexión, en una reflexión a la segunda potencia, por el cual el pensamiento tiende a la recuperación de una intuición que por el contrario se pierde, de cierto modo, en la medida en que se ejerce ${ }^{22}$.

De esta forma, Marcel dejó claro que el orden del misterio no es ininteligible, sino, al contrario, permite la comprensión de una intuición que tiende a perderse cuando se ejerce el pensamiento 23 . El misterio, por lo tanto, invita a recuperar esta "intuition aveugle" 24 mediante el ejercicio de la reflexión segunda sobre ciertas experiencias que lo transparentan. El misterio es, pues, inteligible, pero no totalmente abarcable, pues la distinción del en mí y el delante de mi pierde significación ${ }^{25}$.

Así, el ser coincide con el interior del hombre, por eso la manera de aproximación a él no es mediante fórmulas deductivas abstractas, sino a través del recogimiento y la existencia concreta. Para Marcel, en el acto del recogimiento, se hace una interpretación como unidad y se toma una actitud frente a la vida desde lo que cada uno es $^{26}$. En ese proceso, el conoci-

21 Marcel considera que tratar al ser como un predicado es objetivarlo. Cf. MEII, 37-42.

22 EAI, 147.

23 «Je n'hésiterai pas à dire qu'il faut aller encore plus loin, et reconnaître que l'acte même de penser est mystère, puisque le propre de la pensée est d'apprendre toute représentation objective, toute figuration d'elle-même, toute symbolisation comme inadéquate ». RI, 95.

24 Marcel utilizó la noción "intuition aveugle" para indicar que no se puede hablar aquí de una intuición propiamente dicha ya que "plus une intuition est centrale, plus elle occupe le fond de l'être qu'elle illumine, moins elle est susceptible précisément de revenir sur soi et de s'appréhender”. PA, 65. Además de ello, la intuición le pareció a Marcel dependiente de los presupuestos gnoseológicos de la reflexión primera, a saber, de la relación sujeto-objeto, porque depende del ver. Cf. $\mathrm{HCH}, 69$. Esto va en contra de la relación sujeto-sujeto que es la propia de la reflexión segunda y de la participación. El peso ontológico de la experiencia, admite posteriormente, está sentado sobre una apercepción y no sobre una intuición. Esa apercepción la entiende como un “visión que reúne”, basándose en la palabra griega suneidesis. Cf. DH, 105-106.

25 Cf. RI, 94.

26 Cf. MEI, 142.145.147-48; EAI, 165-166. 
miento no es un simple "conocer", sino que es un "reconocer"27. Esta es la explicación de por qué el pensamiento de Marcel está tan expuesto en las obras dramáticas y en la música, pues es allí donde suele ser más fácil expresar esa intuición y el tipo de comprensión que obedece al misterio ${ }^{28}$. Una de las formas habituales de explicarlo es cuando decimos, por ejemplo, que comprendemos una melodía, mientras hay otras que quizás no se entienden. La música no se evidencia por los conceptos, sino que es una forma singular de expresión de la interioridad ${ }^{29}$.

El reconocimiento del ser, desde las vías del arte y las relaciones humanas, se vuelve, entonces, el contrapeso a la amenaza de la muerte como el no-ser. ¿Cómo se puede entender esto? Ante todo, como una manera de luchar contra los efectos existenciales que puede conllevar la visión de la muerte como el no-ser. De entre ellas está especialmente la angustia y la desesperación. Es más, desde esta perspectiva, le pareció a Marcel que el mundo se orientaría hacia lo absurdo, pues todo se vería condenado a la destrucción y a la desaparición ${ }^{30}$. Esto lo compara Marcel con la mirada petrificante de la Medusa; gorgona que, según la mitología griega, tenía el poder de petrificar a todo aquel que la miraba.

Las objeciones decisivas en este punto de la reflexión podrían ser ¿por qué habría necesidad de afirmar el ser si al no afirmarlo la muerte deja de significar por consecuencia el no-ser, por lo que sería simplemente un hecho más de la vida, promoviendo así la aceptación de la misma como tal? Aún más, ¿Qué sentido tiene la afirmación del ser si éste no cambia en nada el hecho de la muerte en sí, por lo que de todas maneras el rostro de la Medusa cumpliría con su objetivo de desesperación y petrificación? En otras palabras, lo que está en juego en la primera pregunta es la posibilidad de aceptación de la muerte como un hecho del orden natural sin necesidad de una apelación metafísica. La segunda consiste en que la afirmación del ser no cambia en nada el hecho de la muerte en sí, por lo tanto sería innecesario, desde un punto de vista existencial, el desarrollo de esta ontología.

Lo primero a tener en cuenta es que el desarrollo de una metafísica no fue para Marcel una forma premeditada de contraponer el ser a la muerte. Llega a la noción de ser por su interés en el hombre concreto y por darse cuenta de la insuficiencia de la filosofía idealista y del pensamiento científico para la comprensión de la persona y sus interrelaciones. En otras palabras, considera que en el encuentro con el otro hay una necesidad de hablar de $\operatorname{ser}^{31}$.

27 Esto debido a que el ser no se puede entender como un dato: « Si maintenant nous nous attachons à l'être entendu comme innépuisabe concret, nous devrons observer d'abord qu'il ne peut pas à proprement parler faire fonction de donné, qu'il ne peut pas être constaté, mais seulement reconnu ». RI, 96.

28 Marcel, además de filósofo, fue dramaturgo y músico. Él representó en la siguiente metáfora toda su obra intelectual: "Mi obra puesta en su conjunto me parece poder ser comparada con un país como Grecia que contiene a la vez una parte continental y una parte insular. La parte continental son los escritos filosóficos, aquí me encuentro, de alguna manera, al lado de otros pensadores de nuestro tiempo como Jaspers, Buber y Heidegger. Las islas son las piezas de teatro (...). Y si se le puede agregar - no creo que sea ficticio-el elemento que vincula el continente y las islas es la música. La música es verdaderamente la capa más profunda". EPM, 54-55.

29 Marcel expresó que su sensibilidad a la música y, por lo mismo, su ejercitación de la escucha, le permitió traspasar los conceptos puramente espaciales de la mirada. Cf. MARCEL, G., "La musique dans mon œuvre", en LMGM, 100.

30 Es por esto que para Marcel el valor está unido al ser. Cf. PLOURDE (1985) 541-548 y 559.

31 Por esto se explica su distinción entre "él” y "tú”, según la cual, "él” es aquel con quien se tiene comunicación pero no comunión, mientras que el "tú" es aquel con quien se tiene una relación más íntima y personal. Cf. EAI, 130-132. Esta distinción no es subjetiva sino intersubjetiva, pues consiste en la comunicación en tanto 
Marcel, desde pequeño, tuvo una conciencia especial del lazo que lo unía a los otros y de la dignidad de este vínculo. Debido a ello es que admite haberse sentido decepcionado en muchas ocasiones cuando los demás no valoraban la participación en su vida propia. No sin razón confiesa esto como una vulnerabilidad, pero a la vez fue lo que le inspiró y le permitió ahondar en la intersubjetividad: "me pregunto incluso si no deja de lado lo esencial; es decir, el hecho de que desde siempre, sí, desde mi primera infancia, he aspirado a sentirme en consonancia con el otro. Esta imagen musical es, creo, la única adecuada" ${ }^{32}$.

Esa consonancia entre personas expresa un peso o un valor en la existencia que abre una posibilidad o una promesa de decapitar a la Medusa, a saber, a ese rostro petrificante que envuelve todo en la desesperación y el sin sentido ${ }^{33}$. Por esa necesidad del otro, para Marcel, la Medusa amenaza especialmente con la pérdida del ser amado. La muerte del ser amado se convierte así en la evidencia más fehaciente de la capacidad que tiene la muerte para vaciar toda la existencia de sentido. Por eso, en una conferencia llamada Présence et Immortalité, Marcel dejó constancia que el punto crucial en su pensamiento es, precisamente, la cuestión del amor y la muerte:

No dudo en decir que mi vida sin más -y la vida misma de mi espíritu- se ha desarrollado bajo el signo de la muerte del otro, y aquí está el origen lejano de la controversia que me hizo discutir con León Brunschvicg, en el Congreso Descartes en 1937: cuando me reprochó conceder más importancia a mi propia muerte que la que él le atribuía a la suya propia; yo le respondí sin vacilar: "lo que cuenta, no es mi muerte, ni la suya, es la de quienes amamos." En otros términos, el problema, el único problema esencial, es el que suscita el conflicto entre el amor y la muerte. Si hay una certeza inquebrantable, es que un mundo abandonado por el amor no puede más que ir a parar en la muerte, pero también es allí donde el amor persiste, allí donde triunfa sobre todo lo que tiende a degradarlo; la muerte, en definitiva, no puede no ser derrotada ${ }^{34}$.

En este texto, Marcel presenta una estrecha relación entre el amor, el ser, la muerte y la inmortalidad que reúne la esencia del asunto que nos ocupa. Según Marcel, la muerte no puede no ser vencida porque es justo contra ella que el amor persiste, lo que sería un indicio de inmortalidad, aunque nunca una prueba. Esto es así porque, a través de la vivencia del

que seres, no en tanto que cosas. El pensamiento humano tiene la posibilidad de objetivar, pero en el trato con el "otro", especialmente con quien se entra en comunicación íntima, ese pensamiento consta de otras características, la principal de ellas es que quien habla conmigo es un "ser", es decir no es una "cosa". Cf. MEII, 155 .

32 ECD, 21.

33 En Présence et Immortalité, afirma al respecto que: "On peut dire en gros que je me suis proposé dans mon œuvre de chercher s'il est possible sans retomber dans le mensonge de résister à cette fascination, de décapiter la méduse. Je dirai assez volontiers dans ce sens que si le héros mythologique type pour Sartre est Oreste, pour moi il me semble que c'est Persée. Je dis ceci en gros et sans me demander ce que c'est qu'Andromède". PI, 181. Si para Sartre, el hombre encuentra la libertad unida a la soledad en la figura de Orestes, en la figura de Perseo, Marcel encuentra la libertad unida a la comunión. Si por el primero entra la nada a la existencia, por el segundo, se reconoce el ser en la existencia.

34 PI, 182. 
amor, se descubre el ser como presencia ${ }^{35}$. No obstante, ese amor no puede ser ni voluntarioso ni egocéntrico, pues trasciende la voluntad y el cálculo racional. De esta forma, el amor llena de peso ontológico a la existencia, por lo que el ser descubierto en la relación intersubjetiva se convierte en el contrapeso al hecho de la muerte como el no-ser ${ }^{36}$. Esto no se refiere a una inaceptación de la muerte como tal, sino a una explicación parcial de la misma con base en una visión comprensiva del hombre en su integralidad.

Según esto, el tema del ser y la intersubjetividad exigen un replanteamiento de la comprensión de la muerte de acuerdo a la relación sujeto-objeto, para entenderla desde la relación yo-tú, propio del estatuto gnoseológico de la intersubjetividad o del amor. Desde esta reflexión, hay aceptación del hecho de la muerte como tal, pero a su vez hay esperanza $\mathrm{y}$ fidelidad.

Por otro lado, referente a la objeción que indica que la afirmación del ser en nada cambia el hecho de la muerte como tal, hay que decir que una vez se tiene en cuenta todo lo anteriormente dicho, la apertura del conocimiento en la reflexión segunda a estas cuestiones permite, para Marcel, la reivindicación de la libertad frente a la muerte. El propósito de este pensador francés nunca ha sido incidir en el hecho como tal de la muerte, sino en la manera cómo se comprende ésta, para así recrear la relación con la misma, resistiendo a la atracción hacia la desesperación y el sin sentido. Por lo mismo, la manera como existencialmente se posiciona el hombre frente a la muerte se crea a partir de la libertad: "La realidad o el alcance de la muerte no es independiente de la forma en que la considero, debería decir más bien en que la juzgo" 37 . Entonces, de lo que se trata aquí es fundamentalmente de la relación que une al ser humano con su muerte ${ }^{38}$ y no un estudio sobre qué es la muerte ${ }^{39}$.

\section{La muerte como prueba}

Una vez llegados al punto en que la libertad tiene un puesto importante en la reflexión sobre la muerte, se entra en el terreno de la misma como prueba para las relaciones humanas y la existencia como tal. En efecto, Marcel logra ver más allá del rostro petrificante de la

35 El tema de la presencia es el mejor indicio de la inmortalidad. La presencia es la manifestación del ser, la cual se puede percibir sensitivamente. No obstante, la presencia no puede ser tratada por la verificación empírica según la reflexión primera, pues desde este punto de vista se podría reducir a una simple percepción subjetiva. Hay que advertir que la presencia no es una nueva forma de introducir la antigua distinción entre alma y cuerpo, donde la presencia correspondería al alma y ésta sería inmortal. La presencia, por el contrario, depende íntimamente de la intersubjetividad. En ella se revela un centro que es común a todos los hombres y, por el cual, la comunicación trasciende los sentidos y se sitúa en un plano espiritual. De hecho, cuando se da la descentralización en el amor oblativo o, simplemente, cuando se entra en comunicación, lo cual es expresado por Marcel como el paso de un "él” a un "tú”, el otro me revela a mí mismo. Hay, por lo tanto, una participación del uno con el otro. Los dos comparten un centro que les hace más ellos mismos y que se traduce en alegría y plenitud. Por eso es que estos análisis llevan a Marcel al planteamiento del ser. El ser es comparado con una luz que disipa las tinieblas y la oscuridad de la pregunta ¿qué soy yo? Cf. PI, 179-193. Sobre la presencia, ver: GRASSI (2016) y HANLEY (1976).

36 Cf. FTC, 170; PI, 105.

37 PI, 192. También hay referencia en RI, 186-187; EVJ, 86.

38 Cf. FTC, 157.

39 Así lo expresa Marcel cuando advierte que su intención no es hacer algo parecido a una ontología de la muerte. Cf. MEII, 147. 
muerte y encuentra en ella una oportunidad para el reconocimiento de lo más valioso del ser humano. La muerte como prueba de la existencia estriba básicamente sobre el reconocimiento del ser en el sentido ya expuesto, a saber, en la opción por la existencia ante la fascinación que la muerte como el no-ser ${ }^{40}$.

La prueba hace referencia explícitamente a algo que está en juego, algo que debe mostrar su valía o su existencia. Se entiende implícitamente que quien es probado posee libertad para optar. Esa libertad, cuando afirma al ser, es una respuesta a la llamada del mismo ser, por lo tanto se trata de un reconocimiento. De ahí que bajo la noción de ser Marcel hable de la exigencia ontológica ${ }^{41}$ como una vocación ${ }^{42}$. La libertad, en este punto, no es autonomía ${ }^{43}$, tampoco se reduce a la capacidad de escoger con indiferencia ${ }^{44}$, sino que "lo propio del acto libre consiste en que contribuye a hacerme lo que soy" 45 .

De acuerdo con esto, el hombre posee la facultad de elegir entre negar/negarse o invocar/afirmar el ser y su perennidad. Esta afirmación es propia de la ontología: "Problema de la afirmación. Toda la ontología se concentra bajo el acto de afirmar, considerado, a decir verdad, no ya en sí como acto, sino en su intencionalidad específica" ${ }^{\text {"L }}$. La afirmación nunca genera la realidad, sino al contrario. La reflexión segunda ahonda en la afirmación en sí.

La necesidad de esa afirmación e invocación aparece notablemente en el sufrimiento. Ante él, siempre está la posibilidad de dejarse llevar por la desesperación y la angustia o, por el contrario, de poder tomar el camino de la esperanza ${ }^{47}$. Por eso Marcel expresa que "la esperanza se sitúa bien en el cuadro de la prueba, puesto que no sólo le corresponde, sino que constituye para ella una verdadera respuesta del ser"48. La muerte inevitable provoca un cambio que consiste en pasar de una resistencia a la enfermedad a un entregarse con un sentido nuevo.

Para dar respuesta positiva al sufrimiento y a la enfermedad, el hombre sólo tiene que reconocer la necesidad del otro para la constitución de sí mismo, especialmente del ser por quien se entrega la vida. Marcel considera que esta vía está muy unida a la del hombre considerado santo, quien sale del egocentrismo y se centra en la alteridad:

Lo que he percibido en todo caso es la identidad oculta del camino que conduce a la santidad y del camino que conduce al metafísico a la afirmación de ser; la necesidad,

40 La muerte como no-ser que provoca en el ser humano el sinsentido de su existencia, termina por desconocer el ser y evitar que se le reconozca y se le realice en la existencia. Aquí está la diferencia entre ser y existencia, y es que el primero trae consigo un estado de plenitud, mientras que la segunda sólo hace referencia a lo que es real. Cf. PLOURDE (1985), 215.

41 La cuestión del ser en Marcel se vuelve "exigencia del ser”, porque el análisis inicia en lo cotidiano, de donde resalta esta exigencia. Es decir, no es la construcción de un concepto abstracto, sino la respuesta a una necesidad constante del hombre. Cf. EAI, 155-156; PA, 50-51; MEII, 60-62.

42 Cf. HV, 28; MEII, 39; EAI, 156.

43 Cf. EAI, 219-220.

44 Cf. MEII, 116-117.

45 MEII, 118.

46 EAI, 175.

47 La muerte permite volcarse hacia el ser, pero también hacia lo que evidencia ese ser, como son la intersubjetividad, la esperanza y la caridad: los acercamientos concretos a través de los cuales se devela el misterio ontológico. Cf. EAI, 149.

$48 \mathrm{HV}, 38$. 
sobre todo, para una filosofía concreta de reconocer que hay ahí un sólo y mismo camino. Añado que ahí aparece el significado de la prueba del hombre, en particular de la enfermedad y la muerte y su alcance ontológico. Pero es propio de esta prueba el no poder reconocerla; es una llamada a una capacidad de interpretación y asimilación que coincide con la misma libertad ${ }^{49}$.

Entonces, la santidad, según la entiende Marcel, consiste en el reconocimiento de ese camino ontológico. Una vez se ha dotado de sentido al sufrimiento, basados en la esperanza que hunde sus raíces en lo ontológico, el paso a la afirmación del ser, en tanto exigencia del mismo, suscita la visión de la vida como un sacrificio, como una entrega a los demás ${ }^{50}$. Cuando se da esto, la muerte, entonces, no se presenta como una condena anuladora y demoledora $^{51}$, sino como una prueba de lo más valioso de la vida. Es la vida como prueba.

Marcel llegó a estas apreciaciones sobre la muerte y el ser después de reflexionar por un par de décadas. En especial le tomó tiempo desarrollar el método a través del cual se podía acceder a la cuestión de la muerte desde la perspectiva expuesta. Ese método, como ya se ha indicado someramente, establece parámetros para comprender el derrotero que Marcel siguió en su filosofía y en cómo pensar la muerte. Sin él, no sería posible poner en valor todos los alcances existenciales que tiene la muerte cuando ésta se convierte en una amenaza como el no-ser.

\section{Pensar la muerte}

Después de lo expuesto hasta aquí, es oportuno dedicar un espacio a la manera cómo ha sido el proceso de reflexión de la muerte en la obra de Marcel, especialmente poniendo atención al método gnoseológico, pues la muerte varía de acuerdo a la metodología cognoscitiva con la cual se trate. Por esta razón, Marcel distingue varias formas de pensar e interpretar la muerte. Cada una de ellas de acuerdo a la pregunta y el enfoque que las motive. Como se ha visto, lo primero que llamó la atención del autor sobre el hecho de la muerte fue el qué será de los muertos; en otras palabras el tema de la inmortalidad. Ya que su enfoque es la muerte en relación con la sobrevivencia del ser amado, la pregunta por la relación con su propia muerte -“mi muerte", como expresa Marcel- le parece secundaria, tal cual lo expresó en varias oportunidades, especialmente en el Congreso de Filosofía de 1937 ante León Brunschvicg52. No obstante, la apertura del método de conocimiento que hace para el tratamiento del ser, le permite detallar mejor la relación que une al hombre con

49 EAI, 105.

50 El sacrificio como negación del nihilismo : «Tentation de penser que n'avoir rien c'est n’être rien ; et de fait la pente de vie naturelle, c'est de tendre à s'identifier avec ce qu'on a ; par-là la catégorie ontologique tend à s'anéantir. Mais la réalité du sacrifice est là pour nous prouver en quelque sorte en fait la possibilité pour l'être de s'affirmer comme transcendant à l'avoir ». EAI, 104-105. Para Marcel, el hecho de que sea posible el sacrificio significa un testimonio de la capacidad del hombre de encontrarle sentido a la vida por encima de los intereses egocéntricos y la visión pesimista de la vida. Trascender el tener quiere decir, poder tratar a los demás como un "tú", a saber, establecer con los otros relación de ser a ser, no de ser a objeto. Sólo así se puede dar la vida por alguien. Pues, de hecho, el sacrificio es darse o consagrarse a. Cf. RI, 106.

51 Cf. FTC 159-163.

52 Cf. PI, 182; MEII, 152. 
su propia muerte, haciendo una distinción entre la mirada fenomenológica de la misma y la híper-fenomenológica o metafísica.

En sus primeros escritos, especialmente en Etre et Avoir Marcel ve una clara dificultad al pensar su propia muerte. Esto bajo el primer enfoque que coincide con la cuestión de la inmortalidad. Comenzar con este tema es encontrar forzosamente un aprieto por la necesidad de objetivación que aparece a la conciencia. Por esta época -finales de la década de los veinte- apenas estaba entretejiendo y configurando la distinción entre "problema y misterio" y entre "reflexión primera y reflexión segunda". Debido a ello, siente un conflicto entre sus presupuestos metodológicos que llevan, inevitablemente, a pensar la muerte como el final total, y su experiencia de vida, la cual está acompañada por la presencia de su madre y las relaciones interpersonales que le dan una apercepción profunda del $\operatorname{ser}^{53}$. De acuerdo a esto, expone:

Yo no puedo tratarme en absoluto como término distinto de mi cuerpo, que estaría con él en una relación determinable. Como ya he dicho en otras ocasiones, desde el momento en el que el cuerpo es tratado como objeto de la ciencia, al mismo tiempo me exilio al infinito. Aquí está la razón por la que no puedo pensar mi muerte, sino tan sólo la interrupción del funcionamiento de esta máquina (Illam, no hanc). Quizá sería más exacto decir que no puedo anticipar mi muerte, es decir, preguntar qué seré yo cuando la máquina deje de funcionar ${ }^{54}$.

Estas palabras escritas entre 1927 y 1928, que hacen parte de un comunicado a la Sociedad de Filosofía, tienen como dato esencial el conato de lo que es su antropología de la encarnación. Dentro de las primeras reflexiones sobre ello, se encuentra que yo no puedo separarme de "mi cuerpo", sino sólo por imaginación, por lo que éste adquiere un estatuto metafísico especial, sobre el que se entiende la existencia de las cosas. Sobre esto dice:

Cuando afirmo que una cosa existe es que considero tal cosa como vinculada a mi cuerpo en cuanto susceptible de entrar en contacto con él, por indirectamente que sea. Únicamente es preciso tener muy en cuenta que esta prioridad, que de esta manera atribuyo a mi cuerpo, se debe al hecho de que éste me es dado de modo no exclusivamente objetivo, al hecho de que es mi cuerpo. El carácter, al mismo tiempo misterioso e infinito, de la vinculación entre yo y mi cuerpo (no empleo el término relación a propósito) tiñe, en realidad, todo juicio existencial. Esto viene a significar que no se

53 Fue por ello que en su primer libro Fragments Philosophiques (FP), considera que sólo por el hecho de amar o de ser libres, la muerte queda vencida y se puede afirmar la inmortalidad: « Nous avons déjà vu jusqu'ici que la liberté ne peut poser comme transcendant à elle un fait, une vérité d'elle-même qui serait sa mortalité. Mais alors l'immortalité affirme par la foi n'est pas un fait à proprement parler, elle ne se ramène pas à une survie empirique (cas rien de ce qui a rapport à la existence au sens empirique ne peut être impliqué dans l'ordre de la liberté) ». FP, 82-83. «L'amour enveloppe l'affirmation de la survivance (et nous devons nous affirmer nous-mêmes comme survivants pour autant que nous sommes objet d'amour). L'amour ne crée pas la survivance, mais il en enveloppe l'affirmation ». FP, 84. Estos primeros acercamientos a la inmortalidad desde la fe corresponden a la primera etapa de su pensamiento dónde estaba imbuído del idealismo y del racionalismo. Una vez que crea el método de la filosofía concreta, esas afirmaciones se matizan. Cf. URABAYEN (2001) 723.

54 EAI, 12. 
puede disociar: Existencia, conciencia de sí como existente, conciencia de sí como ligado a un cuerpo, como encarnado. De esto parece derivarse varias consecuencias importantes: En primer lugar, el punto de vista existencial sobre la realidad no parece ser otro que el de una personalidad encarnada; en la medida en que podemos imaginar un entendimiento puro, no hay para este posibilidad ninguna de considerarlas cosas como existentes o no existentes..$^{55}$

Con lo anterior, Marcel rechaza radicalmente el ejercicio mental de Descartes, según el cual el pensamiento se cree desligar del cuerpo para autoafirmarse como una sustancia distinta y convertirse en criterio de juicio, como se lee en el Discurso del Método ${ }^{56}$. Es un paso en contra del "cogito" de Descartes, pues cuando se concibe a éste como "puro", no se puede hablar de la existencia de ninguna cosa. No es difícil, entonces, comprender el por qué le resulta arduo hablar de mi muerte, pues, una vez se le concibe bajo las premisas expuestas, sólo se podría pensar que el cuerpo cesa cuando la máquina inicia su proceso de desaparición. Aquí se está tratando a la muerte desde el hecho como tal, bajo la preocupación de lo que "seré una vez ella ocurra".

Debido a este tropiezo, Marcel entiende que el problema se encuentra en la adhesión al paradigma psicofísico. Éste lleva a la reflexión a entender la muerte como la desaparición absoluta: "Es evidente que si me adhiero a cierto paralelismo psicofísico, me veré tentado a declarar que donde un cuerpo como tal se halla destruido, la misma conciencia queda abolida. Queda por saber qué es lo que se ha de pensar de dicho paralelismo. A mi parecer, la disposición del mundo es tal, que en cierto modo nos vemos expresamente invitados a creer en este paralelismo y, por consiguiente, en la realidad de la muerte" 57.

El método de pensamiento que se efectúa aquí corresponde a la reflexión primera. La muerte es vista desde un punto de vista objetivo, según la relación sujeto-objeto. Como corresponde a los inicios del pensamiento de Gabriel Marcel, el pensamiento busca la verdad como verificación empírica. Se persigue, entonces, una certeza objetiva, según el procedimiento mismo de la ciencia. A través de este proceso, entonces, la muerte aparece simplemente como una desaparición absoluta por cuenta del cese de funcionamiento del cuerpo y su posterior descomposición.

La preocupación por el qué será de la muerte del otro e, indirectamente, conmigo una vez ocurra la misma, finaliza mediante el razonamiento anteriormente descrito. No obstante, la muerte pensada desde la reflexión segunda adquiere nuevos significados que permiten replantear la cuestión. La verdad, en este plano, no se confunde ya con la verificación, sino que se aloja en el ser. Es más, la verdad y el ser se corresponden ${ }^{58}$. La verificación sólo es posible allí donde la cuestión tiene las características de objeto, algo exterior a mí. Por lo tanto, mis sentidos pueden contrastar mi conocimiento con el objeto mismo. Sin embargo, la cuestión del ser, como ya se ha explicado, abre el campo del misterio, en donde dicha verificación es imposible e innecesaria.

55 EAI, 9.

56 Cf. DESCARTES (sf.) 123-124.

57 EAI, 173.

58 Cf. PI, 17. 
La manera como Marcel dilucidó esta nueva forma de tratar la muerte fue pensando en la muerte del otro. De allí que ésta haya sido para él la cuestión más importante, por encima de la pregunta sobre la muerte propia, que sería el tema de la finitud. Para ello parte de la experiencia y de la existencia. Teniendo estos puntos de partida, el conocimiento no versa sobre las cosas exteriores, sino sobre la experiencia de interioridad como ser encarnado ${ }^{59}$. De ahí que a través del recogimiento se proceda a la dilucidación de las cuestiones misterio ${ }^{60}$. La muerte del otro, por lo tanto, especialmente del ser amado, se convierte en un hecho que cobra gran importancia para Marcel porque se puede contar con los datos espirituales - fidelidad, amor y esperanza -, que dan un nuevo marco de comprensión a la cuestión.

La preferencia por la muerte del otro, aparte de lo anterior, está motivada, en Marcel, por su biografía personal, como se ha hecho mención, y también por la imposibilidad de sentir la muerte o tratarla como ya acontecida o aconteciendo ${ }^{61}$. Dicho de otro modo, cuando se vive la muerte de otro, especialmente del ser amado, el conocimiento adquiere un punto de vista existencial ${ }^{62}$. Mientras que desde este enfoque es imposible hacerlo sobre mi muerte. La única opción que tengo para reflexionar sobre mi muerte es hacer una analogía mediante la muerte del otro, para quien su muerte será mi muerte y viceversa ${ }^{63}$. De esa manera, el pensar "mi muerte" no quedaría como simple imaginación.

Ese volcar la reflexión sobre la muerte del otro, está impulsado también sobre las dificultades que conlleva tratar la propia muerte desde un punto de vista fenomenológico, ya que no puede ser considerada como un acontecimiento o un suceso, sino a razón de no tratar al sujeto en cuestión como un ser en situación y encarnado ${ }^{64}$. La reflexión sobre la propia muerte, desbordando la visión fenomenológica de la reflexión primera, la hace Marcel en sus obras de madurez, una vez ha desarrollado el método de reflexión segunda y lo ha aplicado a la cuestión de la muerte del otro. De esta manera se comprende que en su segundo diario metafísico Etre et Avoir, suscriba la imposibilidad de pensar su propia muerte, como se ha citado anteriormente, pero en conferencias y textos posteriores como Mi muerte y yo ${ }^{65} \mathrm{O}$ Muerte e Inmortalidad ${ }^{66}$, trate el tema abiertamente.

59 Para Marcel el estar en situación tiene que ver con la conciencia primera de ser un cuerpo, mi cuerpo, por el hecho de sentir, a través de ella se parte hacia la dilucidación de la interioridad. Cf. MEI, 117-121. 153-154.

60 Cf. EAI, 165-166.

61 Cf. PI, 49.

62 Para Marcel el punto de vista existencial crea una distinción entre muerte pensada y muerte sentida: « Symétrique des positions sur le mal est sur la mort. La mort, en tant qu'elle est mon terme, ne comporte pas d'au-delà et ne peut pas être transcendée. Mais, tant que telle, elle se confond avec le mal. La mort pensée s'opposé à la mort sentie, elle est au contrarie transcendée d'emblée, mais ceci veut dire ici escamotée. Il faut une tierce position - de même pour le mal ». PI, 73.

63 Cf. FTC, 165. "Mi muerte: no es aún un hecho, ¿es una idea? Yo debería, si fuera el caso, poderla definir, tomarla como objeto; sin embargo, esto me imposible. Yo no puedo rebasarla en pensamiento e imaginarla como sucedida más que a condición de ponerme en el lugar de otro que me sobrevivirá y para quien eso que yo llamo mi muerte será su muerte”. RI, 184.

64 Cf. PI, 40.

65 Conferencia que aparece en FTC, 157-173.

66 Conferencia dada en Friburgo de Brisgovia el 4 de junio de 1962. Aparece en el libro Auf der SuchenachWahrheit und Gerechtigkeit, Knecht, Frankfurt, 1964. Con traducción al español como En busca de la verdad y de la justicia, seis conferencias a estudiantes universitarios, Herder, Barcelona, 1967. 
Hablar de reflexión segunda es hablar de hiperfenomenología o metafísica ${ }^{67}$. Esto quiere decir que la cuestión del ser cobra un puesto importante como referencia del camino reflexivo que se haga. El conocimiento que de allí provenga tiene una base existencial que lo distingue del idealismo, cuyo sujeto no existe más que para sus conglomerados de enunciados, pues se refiere al hombre concreto, el hombre de carne y hueso como lo diría Miguel de Unamuno ${ }^{68}$. De esta manera Marcel busca una certeza en su filosofía, pero no es una certeza objetiva, sino existencial:

Una confusión, sin embargo, sigue siendo posible, me arriesgo perpetuamente a perder de vista esta diferencia esencial entre una certeza poseída y una certeza existencial, cuyo carácter busco aquí definir: la certeza poseída es, por esencia, consignable y transmisible, ella no es adherente a lo que la ha obtenido [...] La certeza objetiva tiende a despersonalizarse. Yo no estoy satisfecho más que a condición de poder decir: estoy convencido que. Referencia a una estructura de la que puedo decir indiferentemente que es la de las cosas o la de las ideas. El caso de la certeza existencial es totalmente diferente. No se trata aquí de eliminar al sujeto, sino de transmutarlo. Esto es aún poco claro. Habría que concretizar más en la necesidad de partir de una ilustración imperfecta, por ejemplo de la certeza que hay en el amor que yo siento por otro o que otro siente por mít ${ }^{69}$.

La certeza existencial entendida como la no anulación del sujeto en el enunciado del resultado del método, según el estilo de la certeza objetiva, obedece a la relación yo-tú y la participación del ser. En tanto todos participamos del ser, en el amor, que es el conocimiento perfecto $^{70}$, se tiene esa apercepción del ser del otro de una forma directa. De allí que sea un tipo de conocimiento directo, como el que se da en la contemplación, en el que se advierte el ser a través de una obra musical o en el acto de recogimiento. Allí no hay necesidad de un proceso discursivo, sino que el darse y el reconocerse es propio del ser ${ }^{71}$.

La muerte del otro, entonces, a través de este marco de comprensión, le permite a Marcel desarrollar los acercamientos concretos al tema de la inmortalidad ${ }^{72}$. Marcel para ello, utiliza la ontología de la fidelidad y de la esperanza que fundamentan esa certeza existencial. Pero, además, acude a la presencia del ser como lo inverificable y, por lo tanto, no constatable de su desaparición absoluta, una vez que el ser amado muere. Por todo ello, Marcel prefiere hablar de existencia detenida:

67 Para profundizar ver el artículo de FARGES (2012-2013).

68 Marcel conocía a Miguel de Unamuno. De hecho, habla de cómo ambos comparten la inquietud por la mortalidad. Cf. FTC, 43.

69 PI, 119-120

70 Cf. JM, 63; MEII, 61. Sobre el amor como conocimiento en el pensamiento de G. Marcel, ver: CAÑAS (1988), LOPEZ (2010), RUIZ (2016) y VALDERREY (1976).

71 En el proceso cognoscitivo no cabe la objeción de la imaginación como comprensión subjetiva, pues según Marcel, en este caso, el ser no se hace una imagen del otro, sino que simplemente lo reconoce de acuerdo a la relación que los une como ser. Cf. PI, 65.

72 La inmortalidad, como se ha visto, se puede pensar desde un punto de vista hiperfenomenológico o metafísico. Cf. PI, 40 . 
Por más oscuras e inciertas que sean esas indicaciones, por más dudosas que sean las posibilidades, ellas están destinadas a obligar al espíritu a romper con toda representación objetiva (mejor diría objetivante) de la vida y de la muerte. La existencia del difunto deja de ser vista como una cosa que se deshace, o una máquina que se desmorona. Haciendo intervenir, por ejemplo, la noción de existencia detenida, me preparo, quizás, a concebir más concretamente y más metafísicamente la existencia en suspenso, sin que yo esté todavía, en modo alguno, en medida de pronunciarme sobre eso que este suspenso anuncia o $\operatorname{prepara}^{73}$.

Esa existencia detenida que asegura el no trato de la persona como una cosa, sino como un ser, lo representa bajo la metáfora del silencio. La razón por la cual relaciona la muerte con el silencio es, básicamente, por lo indefinido y lo oculto que lleva en sí mismo este concepto. Al entrar a un lugar silencioso, se percibirá serenidad, pero también expectativa por el que vendrá o el que será. Lo mismo pasa con la muerte. Al asemejarla al silencio, afirma su apariencia: silencio, pues no se sabe que se oculta, ya que el silencio siempre deja la incógnita. Un ejemplo es cuando alguien responde con un silencio a una pregunta, ahí no se puede determinar nada. De acuerdo con esto "se podría decir, muy simplemente en esta perspectiva, que si la muerte es un silencio, no podemos señalar su término, ya que no sabemos lo que ese silencio oculta, lo que protege, lo que quizá prepara. El sofisma -la tradición- consiste en interpretar ese silencio como no existencia, como caída en el no-ser"74.

De esta manera se puede notar cómo el pensamiento de Marcel fue evolucionando del problema de la muerte al misterio de la muerte. Para ello, el método de la filosofía concreta fue primordial, ya que la muerte tratada como un problema acaba por llevar a la comprensión de la muerte como la destrucción del ser. Mientras que por la reflexión segunda, se pueden hacer acercamientos concretos a la inmortalidad, los cuales están basados en una seguridad existencial que se alcanza a través del cultivo de experiencias espirituales como la fidelidad, la esperanza y el amor. La muerte se interpreta así como un silencio que deja abierta la puerta a la interpretación y a la vivencia de la presencia.

\section{Abreviaturas}

DH La dignité humaine et ses assises existentielles

EAI Être et Avoir I, Journal métaphysique (1928-1933)

EAII Être et Avoir II, Réflexions sur l'irréligion et la foi

ECD En camino ¿Hacia qué despertar?

EPM Entretiens Paul Ricœur-Gabriel Marcel

EVJ En busca de la verdad y la justicia: Seis conferencias a estudiantes universitarios

FTC Filosofía para un tiempo de crisis

HCH Les hommes contre l'humain

HV Homo Viator, Prolégomènes à une métaphysique de l'espérance

JM Journal Métaphysique

73 PI, 67.

$74 \mathrm{HV}, 196$. 
LMGM L'esthétique musicale de Gabriel Marcel

MEI Le mystère de l'être: I. Réflexion et mystère

MEII Le mystère de l'être : II. Foi et réalité

PA Position et approches concrètes du mystère ontologique

PI Présence et Immortalité

RI Du Refus à l'Invocation

TM Tu ne mourras pas

\section{Referencias bibliográficas}

ANDERSON, Thomas (2006), "Gabriel Marcel on Personal Immortality", en American Catholic Philosophical Quarterly, Vol. 80, No. 3, pp. 393-406.

BELAY, Marcel (1980), La mort dans le théâtre de Gabriel Marcel, Librairie Philosophique J. Vrin, Paris.

CAÑAS, Jose Luis (1988), “Tema del amor en Gabriel Marcel”, en Religión y cultura, Vol. 34, No 165,1988 , pp. 349-376.

DESCARTES, René,(sf.), Reglas para la dirección del espiritu. Investigación de la verdad por la luz natural. Discurso del método. Meditaciones metafísicas seguidas de las objeciones y respuestas. Conversación con Burman. Las pasiones del alma. Correspondencia con Isabel de Bohemia. Tratado del hombre. Editorial Gredos, Madrid. Traducción de Manuel María Morente.

FARGES, Julien (2012-2013), “ "L'hyperphénoménologique » et le « métaproblématique ». Remarques sur les limites de la phénoménologie dans la pensée de Gabriel Marcel», en Gabriel Marcel et la phénoménologie, Bulletin de l'association «Présence de Gabriel Marcel», 21, p. 23-52.

GARCÍA, Gaspar (1974), "Muerte e inmortalidad en Gabriel Marcel”, en ARBOR Vol. 89, № 348, pp. $19-42$.

GRASSI, Martin (2016), "La dimensión intersubjetiva de la existencia, en la filosofía de Gabriel Marcel", en Nuevo Pensamiento, Vol. 72, No . 270, pp. 147-159.

HANLEY, Katherine Rose (1976), "Réflexions sur la présence comme signe d'immortalité, d'après la pensée de Gabriel Marcel", en Revue Philosophique de Louvain 74, pp. 211234.

LONERGAN, Martín (1975), “Gabriel Marcel's Philosophy of Death”, en Philosophy Today, Vol. 9, $\mathrm{N}^{\circ} 1,1975$, pp. 22-28.

LOPEZ, Fernando (2010), "Una ontología desde el amor", en Facies Domini, Vol. 2, №. 2 , pp. 365-390.

MARCEL, Gabriel, (1946), (3 ${ }^{a}$ Edición), Journal Métaphysique, Gallimard, Paris.

MARCEL, Gabriel, (1949), Position et approches concrètes du mystère ontologique, J. Vrin Éditeur 6, Place de la Sorbonne, Paris.

MARCEL, Gabriel, (1956), (12e éd), Du Refus à l'Invocation, Gallimard, Paris.

MARCEL, Gabriel, (1959), Présence et Immortalité, Flammarion, Paris.

MARCEL, Gabriel, (1963) Le mystère de l'être : I. Réflexion et mystère, Aubier-Montagne, Paris.

MARCEL, Gabriel, (1964) Le mystère de l'être : II. Foi et réalité, Aubier-Montagne, Paris. 
MARCEL, Gabriel, (1964), La dignité humaine et ses assises existentielles, AubierMontaigne, Paris.

MARCEL, Gabriel, (1964) Homo Viator, Prolégomènes à une métaphysique de l'espérance, Aubier-Montaigne, Paris.

MARCEL, Gabriel,(1967) En busca de la verdad y la justicia: Seis conferencias a estudiantes universitarios. Herder, Barcelona. Traducción de Juan Godo Costa.

MARCEL, Gabriel, (1968), Les hommes contre l'humain, Fayard, Paris.

MARCEL, Gabriel, (1968), Entretiens Paul Ricœur-Gabriel Marcel, Aubier-Montaigne, Paris.

MARCEL, Gabriel, (1968), Etre et Avoir I, Journal métaphysique (1928-1933), AubierÉditions Montaigne, France.

MARCEL, Gabriel, (1968), Être et Avoir II, Réflexions sur l'irréligion et la foi, AubierMontaigne, France.

MARCEL, Gabriel, (1971) Filosofía para un tiempo de crisis, Ediciones Guadarrama, Madrid. Traducción de Fabián García-Prieto.

MARCEL, Gabriel, (1980), L'esthétique musicale de Gabriel Marcel, Aubier, Paris.

MARCEL, Gabriel, (2005), Tu ne mourras pas, textes choisis et présentés par Anne Marcel, Arfuyen, Paris.

MARCEL, Gabriel, (2012), En camino ¿Hacia qué despertar?, Ediciones Sígueme: Salamanca. Traducción de Juan Padilla Moreno.

MAYO, Venancio (1978), El conflicto entre el amor y la muerte, Pontificia Studiorum Universitas A S.Thomas Aq. In Urbe, Roma.

PLOURDE, Simone, (1985) Vocabulaire Philosophique, Editions Bellarmin, Montréal,

RIGOL, Joan (2014) Amor més enllà de la mort en l'obra filosòfica de Gabriel Marcel, Publicacions de la Facultat de Filosofia, Universitat Ramon Llull, Barcelona.

RÍOS, Jesús (1999), "Símbolo y sentido. Hermenéutica de la muerte en Marcel”, en Horizontes de la Hermenéutica, Actas, Universidad de Santiago de Compostela, Santiago de Compostela, pp. 558-560.

RUIZ, Sandra (2016), “Traspasar los límites de la muerte: el amor en Gabriel Marcel y Víctor Frankl" en Estudios 116, Vol. XIV, pp. 175-189.

URABAYEN, Julia (2001), "El ser humano ante la muerte: Orfeo a la búsqueda de su amada. Una reflexión acerca del pensamiento de Gabriel Marcel”, en Anuario Filosófico, 34, pp. 701-744.

VALDERREY, Carmen (1976), El amor en Gabriel Marcel, Editorial Prensa Española, Madrid. 